\title{
Gradient prosodic boundary strength in syntactic disambiguation
}

Gregg A. Castellucci ${ }^{1,2, *}$ \& Dolly Goldenberg ${ }^{2,3}$

${ }^{1}$ Neuroscience Institute, NYU Langone Medical Center, New York, NY

${ }^{2}$ Haskins Laboratories, New Haven, CT

${ }^{3}$ Department of Linguistics, Yale University, New Haven, CT

*gregg.castellucci@nyulangone.org

\begin{abstract}
Unlike syntactic structure, prosodic structure is typically thought of as non-recursive, with categorical boundaries delineating constituents within a rudimentary hierarchy. However, several experimental studies have demonstrated that prosodic boundaries are not produced categorically, and listeners are sensitive to these gradient variations in production, therefore suggesting that prosodic structure is more complex than normally assumed. While intriguing, it remains unclear whether such variability in boundary production is linguistically meaningful. Here, we explicitly test whether variability within a single class of prosodic boundary can be used to disambiguate sentences containing coordinative structures having multiple possible meanings. Interestingly, a majority of participants reliably employed gradient differences in pause duration to differentiate these ambiguous stimuli, demonstrating conclusively that listeners are not only able to perceive non-categorical variation in prosodic boundary strength, but that this variation can be linguistically meaningful. These results therefore indicate that prosodic structure may be less rigid than previously thought.
\end{abstract}

\section{Introduction}

The most prolific theories of prosodic structure hypothesize that the prosodic hierarchy is nonrecursive (Nespor \& Vogel, 1986) and composed of strictly layered prosodic units (Selkirk, 1984). Most studies examining the nature of prosodic structure and the syntax-prosody interface have either implicitly or explicitly made these assumptions; for example, the most common prosodic transcription system - Tone and Break Indices (ToBI) (Beckman et al., 2005) incorporates these notions. Under these restrictions, prosodic structure is obligatorily flat and always contains the same number of layers ordered in the same way. For example, using the standard prosodic categories suggested by Beckman \& Pierrehumbert (1986), prosodic words are incorporated into intermediate phrases which are then incorporated into the intonational phrases of an utterance - no recursion or variations in layering order are permitted.

These assumptions regarding prosodic structure make strong predictions about the nature of the boundaries between prosodic units, specifically that: 1) prosodic boundaries should be discrete and categorical, 2) all instances of a single category of boundary should be functionally equivalent, and 3) that any observed variability in boundary strength (e.g. pause duration, temporal or tonal cues) within a category is not perceptible and/or linguistically meaningful. These predictions are supported by an experimental study demonstrating that increasing the strength of intermediate phrase boundaries did not affect listeners' adjunct attachment preferences (Carlson et al., 2001). Further support for categorical prosodic boundaries is largely lacking, and several studies have instead provided evidence that speakers produce boundaries that are highly variable in strength, and that listeners are sensitive to these variations in boundary production (Ladd, 1996). For example, an electromagnetic articulography study by Krivokapić \& Byrd (2012) showed that speakers produce intonational phrase boundaries that vary widely in strength, and that listeners were able to perceive these differences even though they were all exemplars of a single category of prosodic boundary. A study by Ladd (1988) also demonstrated that prosodic boundaries of the same category are produced variably and display 
different phonetic properties - specifically F0 declination reset - depending on their strength. Likewise, another study by Wagner and Crivellaro (2010) showed that listeners were able to disambiguate the structures of mathematical formulae (e.g. $B+C{ }^{*} D$ vs. $(B+C){ }^{*} D$ vs. $B+(C$ ${ }^{*}$ D)) using only relative prosodic boundary strength. This study also demonstrated that responses in similar syntactic disambiguation tasks were correlated with continuously increasing boundary strength that spanned several boundary types, which is surprising given the hypothesis that differences in the perception of prosodic structure should only result from transitioning between discrete boundary categories. Furthermore, several studies investigating the nature of the syntax-prosody interface have found that relative strength between adjacent boundaries of different categories is utilized by listeners in syntactic disambiguation tasks (Carlson et al., 2001; Clifton et al., 2002; Snedeker \& Casserly, 2010), demonstrating that differences in boundary strength beyond simple category membership is linguistically meaningful and sometimes more important than absolute boundary strength.

Though most experimental evidence fails to support the hypothesized categorical, discrete nature of prosodic structure and boundaries, no study has yet demonstrated that continuous differences in boundary strength within a single category of prosodic boundary are linguistically meaningful. This study aims to address this gap in knowledge by specifically testing whether gradient differences in pause duration at intonational phrase boundaries can be used by listeners to differentiate between possible meanings of syntactically ambiguous coordinative structures. By holding all other factors constant - such as tonal events, speech rate, relative boundary strength, boundary category, syntactic structure, etc. - we specifically test the hypothesis that within-category variation in boundary strength does not result in linguistically meaningful changes in interpretation.

\section{Methods}

Participants

Sixty-two monolingual speakers of American English participated in this study (36 female; 18-62 years of age, mean of 25.5 years), and received 10 USD as compensation. All participants were naïve to the purpose of the study. Experimental procedures were approved by the Institutional Review Board of Yale University. In addition to completing the experiment, participants also provided demographic information (age, gender, and education level).

\section{Stimuli}

Three sentences comprised of three intonational phrases (IP) were spoken by a trained phonetician (GAC) and recorded in a sound isolation booth:

1) Tell Al, and Bill, and Neil. (AndAnd)

2) Tell Al, and Bill, or Neil. (AndOr)

3) Tell Al, or Bill, and Neil. (OrAnd)

(IP boundaries at punctuation)

Each IP boundary was produced with a low boundary tone and followed by an audible pause, and the final phoneme of each intonational phrase was kept constant in order to limit potential segmental differences in phrase-final lengthening. To systematically vary IP boundary strength, each of the original pauses in the 3 sentences were varied in $40 \mathrm{~ms}$ steps from 200 to $440 \mathrm{~ms}$ using Praat (Boersma, 2001) while keeping the other pause constant at $200 \mathrm{~ms}$, therefore yielding 11 stimuli per sentence (Figure 1a). The lower limit of $200 \mathrm{~ms}$ was selected in order to unambiguously signal a major prosodic boundary in all stimuli, as previous research has demonstrated that pauses become perceptible at this duration (Walker \& Trimboli, 1982). 


\section{Stimulus and Response Design}

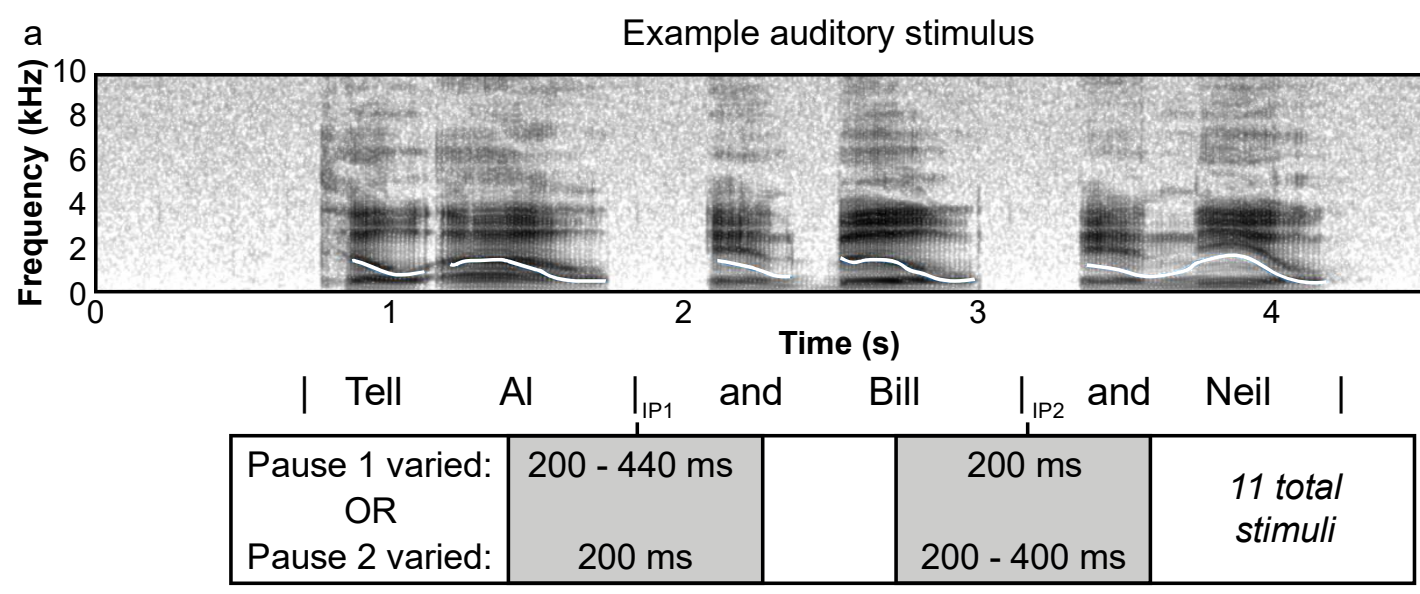

b

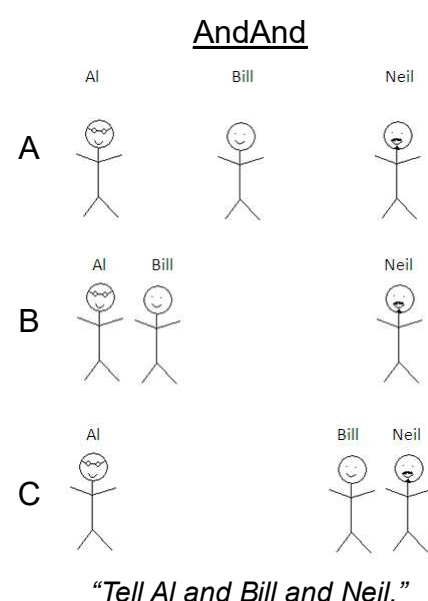

Visual responses

AndOr $\quad$ OrAnd
A

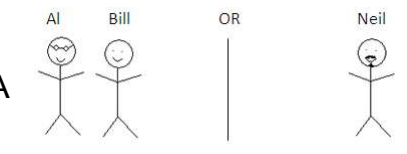

$B$

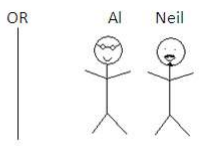

“Tell Al and Bill or Neil.”

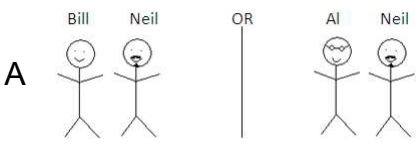

$B \overbrace{}^{A 1}$

"Tell Al or Bill and Neil."

Figure 1. Stimulus and response design. (a) (top) Example spectrogram of AndAnd stimuli with continuous pitch (F0) estimates overlaid in white. In this example, the first and second pauses (at IP 1 and $\mathrm{IP}_{2}$, respectively) both have a duration of $200 \mathrm{~ms}$. (bottom) A schematic demonstrating the generation of the 11 stimuli for each sentence type: while the duration of one pause is held constant at $200 \mathrm{~ms}$, the duration of the other pause is varied in $40 \mathrm{~ms}$ steps from 200 to $440 \mathrm{~ms}$. (b) The visual responses presented to the participants which represented the possible meanings of the auditory stimuli.

Task

Participants were seated in front of a computer wearing headphones (Sennheiser, Wedemark, Germany). Using a custom Praat script, participants were presented with stimuli blocked by sentence type (AndAnd, AndOr, or OrAnd). Task block order was randomized. For each task block, participants were presented with one of the 11 auditory stimuli in randomized order and were instructed to choose the visual response (stick figures representing 'Al', 'Bill', and 'Neil') that best represented the meaning of the sentence they heard (Figure $1 \mathrm{~b}$ ). Participants could replay the auditory stimuli once and were free to move on to the next trial at their own pace. Each stimulus was presented only once in order to avoid habituation and fatigue. A similar forced choice paradigm with visual responses was shown to be successful in eliciting attachment preferences in previous study examining the role of prosodic structure in syntactic disambiguation (Snedeker \& Trueswell, 2003). 


\section{Data Analysis}

Responses to AndAnd, AndOr, and OrAnd stimuli were first arrange by relative pause duration (the duration of the first pause subtracted by second). Any participant who did not respond to a set of stimuli by selecting each visual response at least twice was considered 'nonresponsive' and was excluded from further behavioral analysis (e.g. responding with "A" zero or one times in the AndOr block). Next, the responses of each 'responsive' participant were fit with either a multiple linear regression (AndAnd) or multinomial logistic regression (AndOr and OrAnd) model with relative pause duration and stimulus presentation order as predictors. The observed fit coefficients for pause duration and order were assessed for significance by comparing them to analogous values resulting from fits of each participant's shuffled responses (therefore conserving the biases of each individual). Either paired t-tests or rank-sum tests were used to assess for significance between observed and shuffled values after testing for normality in these data sets with Lilliefors normality tests (see Results for more details). Where applicable, the Sidak correction was used to control of multiple comparisons. All statistical analyses were performed in Matlab (Natick, USA).

\section{Results}

\section{General Responses}

Across all participants, 40/62 participants responded differently to the AndAnd stimuli, 48/60 (data from two participants were lost) responded differently to the AndOr stimuli, and 50/62 responded differently to the OrAnd stimuli. In most cases, nonresponsive participants did not respond differently to more than one set of stimuli (Figure 2a).

\section{Characteristics of Responding and Nonresponding Participants}

a Nonresponder distribution

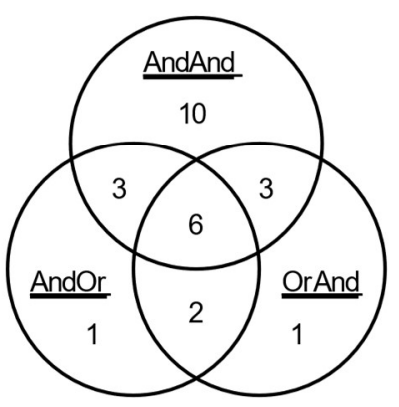

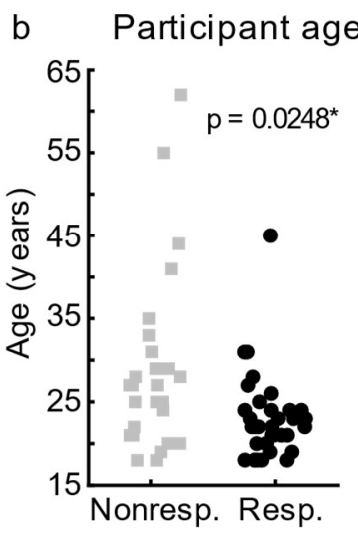

c $P$

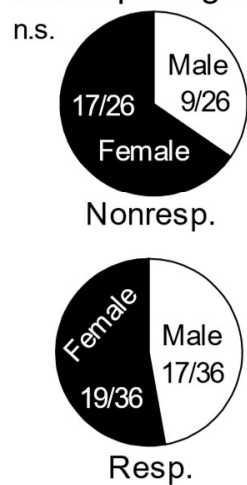

d Participant educatior

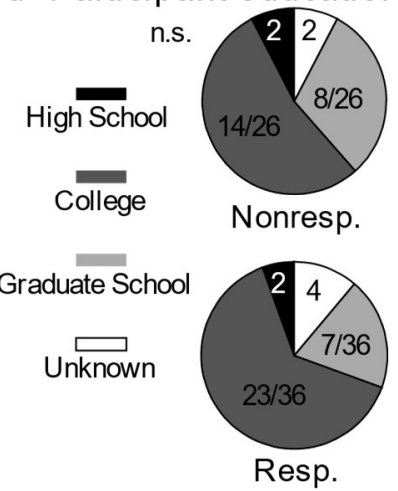

Figure 2. Characteristics of Responding and Nonresponding Participants. (a) Venn diagram illustrating the distribution of nonresponding participants for each task. (b) The distribution of age for both responding and nonresponding participants. Pie charts illustrating (c) gender and (d) education levels for both responding and nonresponding participants. In (b-d), the p-value (or n.s., if not significant) for the multinomial logistic regression coefficients of participant demographics is noted on each plot. In (d), education level denotes the highest level achieved or partially completed.

To determine whether participant age (Figure 2b), gender (Figure 2c), or education level (Figure $2 \mathrm{~d}$ ) was correlated to task responsiveness, a multinomial logistic regression model was fit to responder status with age, gender, and education as predictors (any participant that did not responding differently to any of the 3 sets of stimuli were considered nonresponsive for this analysis [ $n=26 / 62]$ ). Age was determined to be a significant predictor ( $p=0.0248)$, with younger participants being more likely to be responsive than older participants (median age of 
22 years and 27 years for responsive and nonresponsive participants, respectively). Gender ( $p$ $=0.4624)$ and education level $(p=0.8593)$ were not significant predictors of responder status.

\section{AndAnd Task}

Forty of 62 participants responded to the AndAnd stimuli with at least two selections of each possible response (Figure 3a). When pauses were of similar duration, responsive participants tended to group each stick figure individually but when pauses were of considerably different magnitudes, stick figures would be separated into groups delineated by the larger pause (Figure $3 b)$. To assess whether this trend was statistically significant, each responsive participant's responses were fit with a multiple linear regression model with relative pause duration and stimulus presentation order as predictors. Despite the limited sample size available within individual participants ( $n=11$ stimulus presentations), pause duration was a significant predictor of response in $30 / 40$ participants ( $p<0.05$, Sidak correction for multiple comparisons), but was not a significant predictor of shuffled response in any of the responsive participants. Stimulus order was not a significant predictor of actual or shuffled pause duration in any of the responsive participants.

\section{AndAnd Responses}

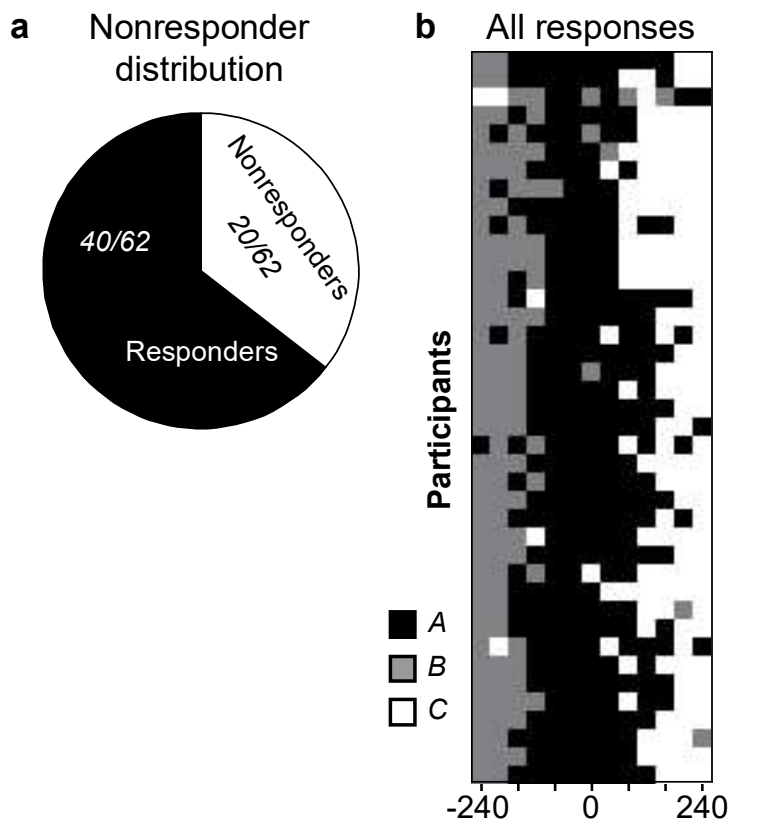

Relative Pause Duration (ms) c Pause duration d

d $\quad$ Order

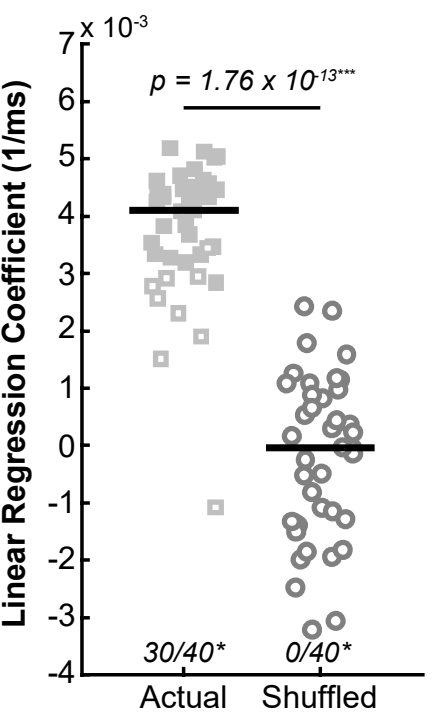

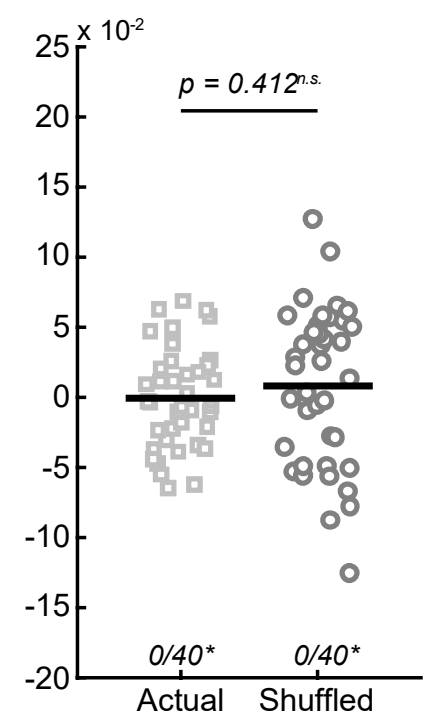

Response Type

Figure 3. AndAnd Responses. (a) Pie graph depicting the number of responsive and nonresponsive participants for the AndAnd stimuli. (b) Colorplot showing the responses of each responsive participant in the AndAnd task arranged by relative pause duration (duration of pause 1 subtracted by the duration of pause 2). Coefficients for (c) pause duration and (d) stimulus order predictors from the multiple linear regression model for task response. Median values are indicated for the actual responses and shuffled responses, and exact p-values (rank-sum test) for comparisons between actual and shuffled responses are indicated on each plot. Likewise, the number of participants whose actual and shuffled responses were significantly predicted by pause duration or stimulus presentation order is noted at the bottom of the plots and denoted by filled points in (c) and (d), respectively $(p<0.05$, Sidak correction for multiple comparisons).

Next, to assess whether pause duration and/or stimulus order significantly predicted response across the cohort of responsive participants, we compared the fit coefficients for pause duration 
and stimulus order to analogous coefficients from fits of each participant's shuffled responses. The pause duration coefficient distribution was normally distributed for shuffled responses ( $p>$ 0.5 , Lilliefors test) but not for actual responses $(p=0.0404)$, so a rank-sum test was used to assess significance. Actual pause duration coefficients were significantly larger than coefficients for shuffled values ( $p<0.0001$, rank-sum test), demonstrating the pause duration was significantly better predictor of response than would be observed if the participants were simply guessing (Figure 3c). For stimulus order, coefficient distribution for both the actual and shuffled responses were normally distributed ( $p>0.5$ and $p=0.1252$, respectively, Lilliefors test), so a paired t-test was used to assess for significance. Fit coefficient values for actual stimulus order was not significantly different from the coefficients from the fits of shuffled responses, confirming the stimulus order was not predictive of response across participants in the AndAnd task (Figure 3d).

\section{AndOr Task}

Forty-eight of 60 participants responded to the AndOr stimuli with at least two selections of each possible response (Figure 4a). Responsive participants tended to have individual biases regarding the meaning of AndOr stimuli when the first and second pauses were of similar duration (e.g. some participants selected ' $A$ ' more often than 'B'). However, when the differences in pause duration were more extreme, responsive participants tended to group the two IPs separated by the smaller duration pause into a single syntactic structure, thus disambiguating the two possible meanings of the stimuli. (Figure $4 \mathrm{~b}$ ). For example, when the second pause was longer than the first, the following structures tended to be perceived:

Prosodic structure:

Syntactic structure: $\left((\text { Tell Al })_{\mid \mathrm{P} 1}(\text { and Bill })_{\mid \mathrm{P} 2}\right)_{\times}(\text {or Neil })_{\mathrm{PP} 3}$

[Tell [[Al and Bill $]_{\mathrm{NP}}$ or [Neil $\left.\left.]_{\mathrm{NP}}\right]_{\mathrm{NP}}\right]$

where ' $x$ ' is an unspecified prosodic constituent occurring at the second pause composed of IP 1 and $\mathrm{IP}_{2}$. To assess whether this trend was significant, each responsive participant's responses were fit with a multinomial logistic regression model with relative pause duration and stimulus presentation order as predictors. Unlike the AndAnd condition where the responses of no participants were significantly predicted by stimulus presentation order, stimulus order was found to be a significant predictor of actual responses in 14/48 participants and a significant predictor of shuffled responses in $1 / 48$ participants $(p<0.05$, Sidak correction for multiple comparisons). However, even after controlling for stimulus order - and despite the limited sample size available within individual participants - pause duration was found to be a significant predictor of AndOr task response in 14/48 participants $(p<0.05$, Sidak correction for multiple comparisons) and a significant predictor of shuffled responses in only 1/48 participants.

To assess whether pause duration and/or stimulus presentation order significantly predicted response across the responsive participant cohort in the AndOr task, we compared the fit coefficients for pause duration and order predictors to analogous coefficients fit to each participant's shuffled responses. The distribution of fit coefficients for pause duration and stimulus order to actual and shuffled responses were found to deviate significantly from normal (all four distributions $p<0.001$, Lilliefors test), therefore rank-sum tests were used to compare actual and shuffled values. Across responsive participants, logistic regression coefficient magnitude for pause duration was significantly higher for actual responses compared to shuffled responses ( $p<0.0001)$, again demonstrating that pause duration was a significantly better predictor of response than would be observed if participants simply chose a response at random (Figure 4c). Conversely, stimulus order fit coefficients for the actual responses were not significantly different from those for the shuffled responses $(p=0.595)$, therefore order was not a significant predictor of response across responsive participants in the AndOr task (Figure 4d). 


\section{AndOr Responses}

a

Nonresponder

distribution

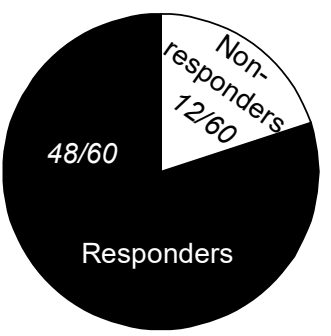

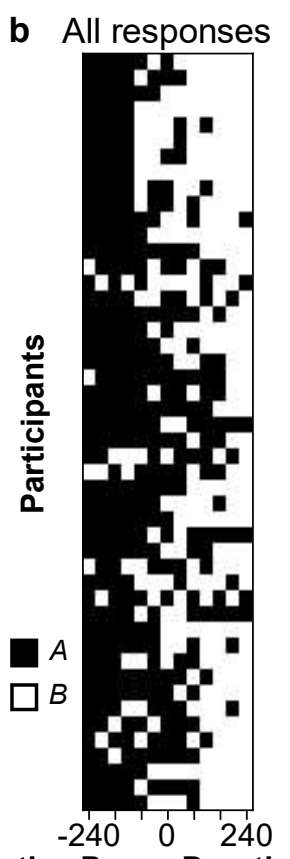

Relative Pause Duration (ms) c Pause duration d

Order

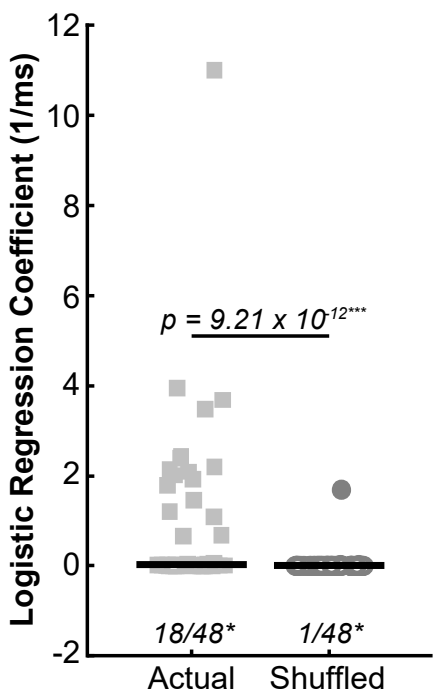

Response Type

Figure 4. AndOr Responses. (a) Pie graph depicting the number of responsive and nonresponsive participants for the AndOr stimuli. (b) Colorplot showing the responses of each responsive participant in the AndOr task arranged by relative pause duration (duration of pause 1 subtracted by the duration of pause 2). Coefficients for (c) pause duration and (d) stimulus order predictors from the multinomial logistic regression for response. Median values are indicated for the actual responses and shuffled responses, and exact $p$-values (rank-sum test) for comparisons between actual and shuffled responses are indicated on each plot. Likewise, the number of participants whose actual or shuffled responses were significantly predicted by pause duration or stimulus presentation order is noted at the bottom of the plots and denoted by filled points in (c) and (d), respectively ( $p<0.05$, Sidak correction for multiple comparisons).

\section{OrAnd Task}

Fifty of 62 participants responded to the OrAnd stimuli with at least two selections of each possible response (Figure 5a). Like in the AndOr task, participants tended to display individual biases regarding the meaning of the stimuli when the two pauses were of similar duration. Also congruent with the AndOr task, when differences in pause duration were larger, responsive participants tended disambiguate the sentences by grouping the two IPs delineated by the shorter pause duration into a single syntactic structure (Figure $5 \mathrm{~b}$ ). To assess whether this trend was significant, each responsive participant's responses were fit with a multinomial logistic regression model with relative pause duration and stimulus presentation order as predictors therefore repeating the analysis performed for the AndOr stimuli. Similar to what was observed in the AndOr task, stimulus presentation order was a significant predictor of response in 9/50 participants ( $p<0.05$, Sidak correction for multiple comparisons) but was not a significant predictor of shuffled response in any of the responsive participants. After controlling for stimulus order, pause duration was a still significant predictor of response in $14 / 50$ participants $(p<0.05$, Sidak correction for multiple comparisons), but not a significant predictor of shuffled response in any of the responsive participants. 


\section{OrAnd Responses}

a

Nonresponder

distribution

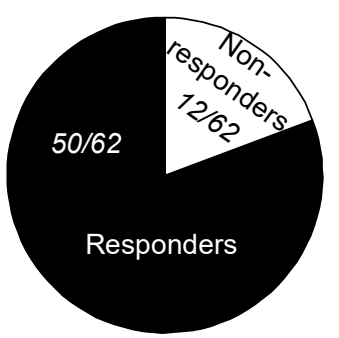

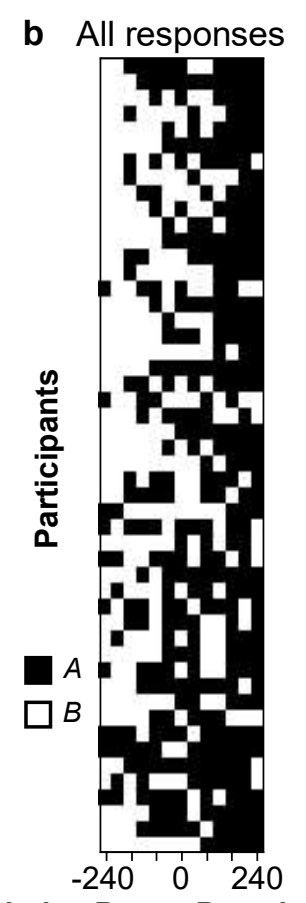

Relative Pause Duration (ms) c Pause duration d

Order
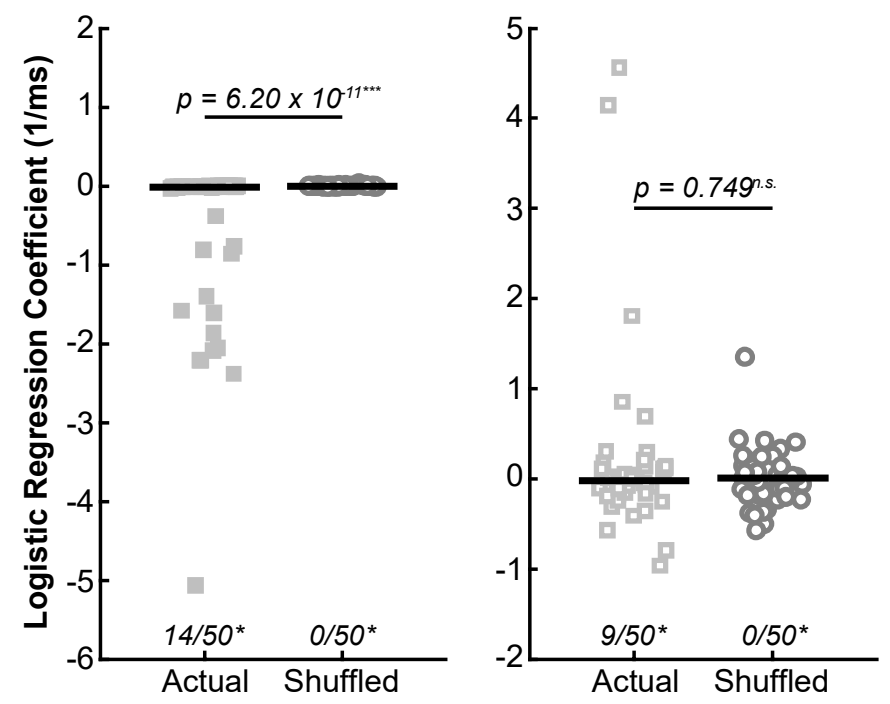

Figure 5. OrAnd Responses. (a) Pie graph depicting the number of responsive and nonresponsive participants for the OrAnd stimuli. (b) Colorplot showing the responses of each responsive participant in the OrAnd task arranged by relative pause duration (duration of pause 1 subtracted by the duration of pause 2). Coefficients for (c) pause duration and (d) stimulus order predictors from the multinomial logistic regression for response. Median values are indicated for the actual responses and shuffled responses, and exact p-values (rank-sum test) for comparisons between actual and shuffled responses are indicated on each plot. Likewise, the number of participants whose actual and shuffled responses were significantly predicted by pause duration or stimulus presentation order is noted at the bottom of the plots and denoted by filled points in (c) and (d), respectively $(p<0.05$, Sidak correction for multiple comparisons).

Finally, to assess whether pause duration and/or stimulus order significantly predicted responses in the OrAnd task, we again compared logistic fit coefficients for pause duration and stimulus order to analogous fits to shuffled responses. The distributions of fit coefficients for pause duration deviated from normal for both actual and shuffled responses $(p<0.001$ and $p=$ 0.0071 , respectively, Lilliefors test), as did the distributions of fit coefficients for stimulus order for both actual and shuffled responses ( $p<0.001$ and $p=0.0115$, respectively, Lilliefors test); therefore rank-sum tests were used to compare actual and shuffled values. Across the 50 responsive participants, logistic regression coefficient magnitude for pause duration was significantly lower for actual responses compared to shuffled responses $(p<0.0001)$ (Figure $5 c)$, mirroring what was observed in the AndOr task but with an opposite sign as the responses were reversed relative to pause duration in the OrAnd task. Therefore, pause duration was again found to be a significantly better predictor of response than would be observed if participants simply guessed at random. Finally, congruent with the AndAnd and AndOr tasks, stimulus order fit coefficients for the actual responses were not significantly different from those for the shuffled responses $(p=0.749)$, therefore order was not a significant predictor of response across responsive participants in the OrAnd task (Figure 5d). 


\section{Discussion}

In summary, we tested whether listeners could make use of gradient differences in pause duration at IP boundaries to disambiguate coordinative structures with multiple possible meanings. In all three tasks (AndAnd, AndOr, OrAnd), a majority of participants parsed the ambiguous sentences by placing the larger syntactic boundary at the prosodic boundary where the longer pause occurred, with this trend being most consistent when the two pauses differed the most in duration. Furthermore, in all tasks, pause duration - but not stimulus order - was a significantly better predictor of participant responses than shuffled responses (i.e. if the participants chose a response at random). Therefore, responsive participants employed gradient differences in IP boundary strength as a syntactic disambiguation strategy, which unequivocally demonstrates that continuous variation in within-category boundary strength can indeed be linguistically meaningful.

Our results conflict with those reported by Carlson et al. (2001), who found that stronger intermediate phrase boundaries did not change adjunct attachment preferences in comparison to weaker intermediate phrase boundaries. The precise reasons for this incongruency are unknown but may be due to methodological differences. Specifically, the stimuli used by Carlson et al. (2001) were natural productions of sentences that incidentally varied in boundary strength but were not manipulated to isolate and continuously vary specific aspects of boundary strengthening, as was performed here for pause duration. Therefore, the range of boundary strengths offered by their naturally produced utterances may have been insufficient to elicit the perceptual changes we observed in this study. In order to investigate this hypothesis, a method similar to the one employed here - which samples a continuous, wide range of boundary strengths - should be adapted to examine intermediate phrases, thus yielding a directly comparable dataset. Regardless, we conclusively demonstrate that variability in IP boundary strength can affect the perception of prosodic and syntactic structure.

It is intriguing that perceptual changes in syntactic structure were observed in this experiment although no categorical variation occurred in prosodic structure of the auditory stimuli. Specifically, IP boundaries - which are typically hypothesized to be functionally equivalent delineated the constituent noun phrases (NP) of the coordinative structures; however, these IP boundaries were perceived as categorically different when the disparity in pause duration between the two boundaries was large. This change in the perception of prosodic structure and consequently syntactic structure - while both boundaries in question are members of the same category can be accounted for in three ways: 1) IP boundaries become a new type of boundary at a certain pause duration, 2) IP boundaries gain additional structure as boundary strength increases continuously, or 3 ) prosodic structure is not categorical in nature.

While theoretically possible, positing a new type of prosodic unit and associated boundary above the IP is problematic as - unlike intermediate phrases and IPs, which are differentiated categorically by tonal events (e.g. Beckman et al., 2005) - this new unit would have no unique features, and instead would be differentiated from IPs solely by a continuous difference in pause duration at its boundaries. This shortcoming can be avoided by hypothesizing that the IP boundaries perceived as a major syntactic boundary in this experiment remained IP boundaries, but gained additional structure not possessed by their counterparts associated with a minor syntactic boundary which possessed a shorter pause. In this situation, the categorical properties of the IP are retained, and the increased structure could be signalled by a longer pause duration. Additional structure could be specifically attained if a restricted degree of recursion is permitted in prosodic hierarchy; for example, following the suggestion of Ladd (1996), prosodic units of one category could be recursively embedded in other units of the same category (i.e. an 
IP embedded in an IP, in this case). While intriguing, further direct evidence for recursion in prosodic structure is lacking.

Our results could also be accounted for by positing the third possibility - that prosodic structure is not strictly categorical and largely lacks absolute classes of units and boundaries. In such a case, the perception of boundary strength would be signalled by relative differences between boundaries rather than distinct tonal and temporal events. This hypothesis is supported by several previous studies showing that syntactic structure is perceived based on relative differences between two prosodic boundaries, and not the categories of the boundaries - for example, a sentence containing two IPs is perceived as having the same structure as one containing two intermediate phrases (Carlson et al., 2001; Clifton et al., 2002; Snedeker \& Casserly, 2010; Wagner \& Crivellaro, 2010). Therefore, in this experiment, the IP with the larger pause duration could have been perceived as signalling the major syntactic boundary solely because it was larger in magnitude than the other IP in the utterance (as indicated by pause duration). However, it is important not to extend this proposal to the extreme and equate all prosodic boundaries as there is substantial evidence that the tonal and temporal events comprising boundaries above the prosodic word are planned (reviewed in Krivokapić, 2014) and that major prosodic boundaries are processed distinctly in the brain (Steinhauer et al., 1999). However, further experimental research is required to determine exactly what types and features of boundaries are planned, and how many categories of prosodic boundaries are processed distinctly by the brain.

Finally, it is worth noting that it is unclear why a substantial proportion of participants did not respond differently to the auditory stimuli. The only demographic trait that significantly predicted task responsiveness was age, with older participants being more likely to be nonresponsive. However, the median ages of responsive and nonresponsive participants differed by only 5 years (22 vs. 27 years, respectively), and the age distributions for each group overlapped extensively, as was the case for both participant gender and education level. Additional work is necessary to determine if task responsiveness correlates with some unmeasured demographic trait, dialectal difference, or perceptual process that varies across the population.

\section{Conclusion}

In brief, we found that a majority of participants used gradient differences in within-category prosodic boundary strength to disambiguate sentences with multiple possible meanings. This finding provides strong counterevidence to the hypothesis that prosodic boundaries are discrete and categorical. Furthermore, these results suggest that prosodic structure may not be strictly non-recursive as typically assumed, or instead that the perception of prosodic structure is exclusively driven by relative differences between boundaries. Further research is required to determine whether our findings extend to other types of prosodic boundaries, such as intermediate phrases (Carlson et al., 2001), and a variety of syntactic structures.

\section{References}

Beckman, M., Hirschberg, S., \& Shattuck-Hufnagel, S. (2005). "The original ToBI system and the evolution ToBI framework", in book: Prosodic Models and Transcription: Towards Prosodic Typology, edited by S.-A. Jun (Oxford University Press, Oxford), 9-54.

Beckman, M. \& Pierrehumbert, J. (1986). Intonational structure in Japanese and English. Phonology Yearbook 3, 58-81.

Boersma, P. (2001). Praat: doing phonetics by computer [Computer Program]. Glot Internat. 5:9/10, 341-345. 
Carlson, K., Clifton, C., \& Frazier, L. (2001). Prosodic boundaries in adjunct attachment. J. Mem. Lang. 45, 58-81.

Clifton, C., Carlson, K., \& Frazier, L. (2002). Informative Prosodic Boundaries. Lang. Speech 45:2, 87-114.

Krivokapić, K. (2014). Gestural coordination at prosodic boundaries and its role for prosodic structure and speech planning processes. Phil. Trans. Royal Soc. B 369: 20130397.

Krivokapić, J. \& Byrd, D. (2012). Prosodic Boundary Strength, an Articulatory and Perceptual Study. J. Phon. 40, 430-442.

Ladd, R. (1996). Intonational Phonology. (Cambridge University Press, Cambridge).

Ladd, R. (1988). Declination "reset" and the hierarchical organization of utterances. J. Acoust. Soc. Am. 84, 530-544.

Nespor, M. \& Vogel, I. (1986). Prosodic phonology. (Foris Publications, Dordrecht).

Selkirk, E. (1984). Phonology and syntax, the relation between sound and structure. (MIT Press, Cambridge).

Snedeker, J. \& Casserly, E. (2010). Is it all relative? Effects of prosodic boundaries on the comprehension and production of attachment ambiguities. Lang. Cog. Proc. 25:7-9, 1234-1264.

Snedeker, J. \& Trueswell, J. (2003). Using prosody to avoid ambiguity: Effects of speaker awareness and referential context. J. Mem. Lang. 48, 103-130.

Steinhauer, K. Alter, K., \& Friederici, A. (1999). Brain potentials indicate immediate use of prosodic cues in natural speech processing. Nat. Neurosci. 2, 191-196.

Wagner, M. \& Crivellaro, S. (2010). Relative Prosodic Boundary Strength and Prior Bias in Disambiguation. Speech Prosody, 100238.

Walker, M. \& Trimboli, C. (1982). Smooth Transitions in Conversational Interactions. J. Social Pscyhol. 117:2, 305-306. 